\title{
NIFS Atomic Numerical Databases
}

\author{
Izumi Murakami and Takako Kato \\ National Institute for Fusion Science, Toki 509-5292, Japan
}

\begin{abstract}
We have compiled the atomic and molecular numerical databases which are available through internet. The databases provide basic atomic data, such as collisional ionization and excitation cross sections, which are important for modeling and diagnosing astrophysical plasmas.
\end{abstract}

\section{Introduction}

Atomic data are important for modeling and diagnosing astrophysical plasmas. For instance, ion abundances are calculated with ionization and recombination rates, and spectral line intensities are estimated with transition probabilities, excitation rates, and etc. The NIFS atomic and molecular numerical databases have numerical data of cross sections and rate coefficients for collision processes between electron, ion, atom, and molecules. The data compilation was started for elements which are important for fusion plasma in 1970s first (Takayanagi \& Suzuki 1976), but extended for other elements later.

\section{NIFS databases}

The NIFS databases consist of sub databases (DBs) categorized with the atomic processes: "AMDIS" is the DB of electron-impact ionization, excitation, and recombination cross sections and rate coefficients. Compilation of ionization and excitation rate coefficients will start soon; "CHART" is the DB of charge transfer and ionization cross sections of ion-atom/molecule collision; and there are other DBs for molecule collision processes and plasma-material interaction. These DBs are available at

http://dbshino.nifs.ac.jp/ since 1997 and registration is required for using them (free of charge for academic research). Now more than 800 people are resisted and more than half of them are international users from 47 countries.

Atomic data are retrievable and numerical data tables and graphs are displayed in a browser. The data shown in a graph can be compared, which is useful for data evaluation. Figure 1 shows an example of the web page for data search. Data are search-able by element, ionic state, author name, and so on.

Data compilation has been done as international and domestic collaborations with atomic physicists and their help and understandings are important and necessary. We are grateful for all their help in past and future. Feedback such as data needs from the data users is also important for developing the database. 


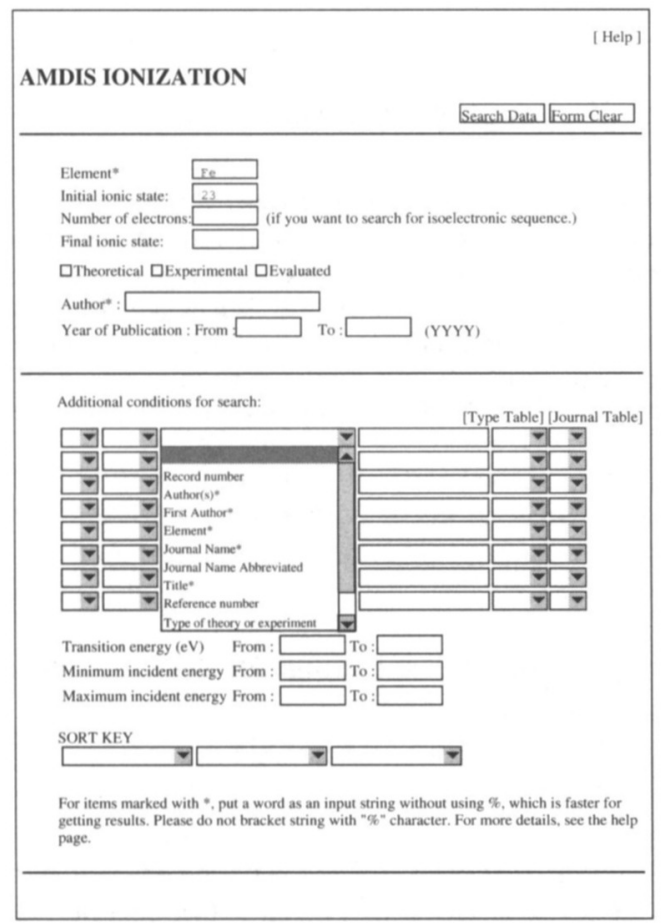

Figure 1. Example of web page image for data search of the NIFS database: AMDIS IONIZATION is the database of electron-impact ionization cross sections.

\section{References}

Takayanagi, K., Suzuki, H. 1976, IPPJ-DT-50 (Inst. Plasma Phys., Nagoya Univ.). 